\title{
The Role of Brain Derived Neurotrophic Factor in HIV-Associated Neurocognitive Disorder: From the Bench-Top to the Bedside
}

This article was published in the following Dove Press journal:

Neuropsychiatric Disease and Treatment

\author{
Henry Michael $\mathbb{1}^{\prime}$ \\ Thabisile Mpofana ${ }^{2}$ \\ Suvira Ramlall $\mathbb{1 D}^{3}$ \\ Frasia Oosthuizen' \\ 'Discipline of Pharmaceutical Sciences, \\ School of Health Science, University of \\ KwaZulu-Natal, Durban, South Africa; \\ ${ }^{2}$ School of Laboratory Medicine and \\ Medical Sciences, University of KwaZulu- \\ Natal, Durban, South Africa; ${ }^{3}$ Department \\ of Psychiatry, University of KwaZulu-Natal, \\ Durban, South Africa
}

\begin{abstract}
Human immunodeficiency virus (HIV)-associated neurocognitive disorder (HAND) remains prevalent in the anti-retroviral (ART) era. While there is a complex interplay of many factors in the neuropathogenesis of HAND, decreased neurotrophic synthesis has been shown to contribute to synaptic degeneration which is a hallmark of HAND neuropathology. Brain derived neurotrophic factor (BDNF) is the most abundant and synaptic-promoting neurotrophic factor in the brain and plays a critical role in both learning and memory. Reduced BDNF levels can worsen neurocognitive impairment in HIV-positive individuals across several domains. In this paper, we review the evidence from pre-clinical and clinical studies showing the neuroprotective roles of BDNF against viral proteins, effect on co-morbid mental health disorders, altered human microbiome and ART in HAND management. Potential applications of BDNF modulation in pharmacotherapeutic, cognitive and behavioral interventions in HAND are also discussed. Finally, research gaps and future research direction are identified with the aim of helping researchers to direct efforts to make these BDNF driven interventions improve the quality of life of patients living with HAND.
\end{abstract} Keywords: neurotrophins, neuroplasticity, HIV/AIDS, cognition, BDNF

\section{Introduction}

About 36.9 million people live with HIV globally. The decline of AIDS related deaths, from a peak of 1.9 million in 2005 to 940,000 in 2018, has been attributed to the global scale up of ART with 21.9 million accessing treatment. ${ }^{1}$ While medical morbidity has significantly improved in the ART era, HIV-Associated Neurocognitive Disorders (HAND) remain common with the prevalence of HAND globally estimated at $50 \%{ }^{2}$ HAND can be categorized in increasing degree of severity into asymptomatic neurocognitive impairment (ANI), mild neurocognitive disorder (MND) and HIV-associated dementia (HAD). ${ }^{3}$ The cognitive domains usually affected are psychomotor skills, speed of information processing, executive function, episodic memory, attention/working memory, language ${ }^{3,4}$ and sensory perception. ${ }^{5}$ These impairments disrupt work and daily activities of people living with HIV (PLWH), impact medication adherence negatively, lowers quality of life and can be linked to mortality independent of viral load. ${ }^{6}$

There is a complex interplay of biopsychosocial factors implicated in the pathogenesis of HAND as summarized in Table 1. These risk factors impact cognition in complex ways by altering mechanisms of neuroplasticity (Figure 1). ${ }^{7}$
Correspondence: Henry Michael Discipline of Pharmaceutical Sciences, School of Health Science, University of KwaZulu-Natal, Westville Campus,

Durban 404I, South Africa

Tel +27732770601

Email doctorprince322@hotmail.com

Neuropsychiatric Disease and Treatment 2020:16 355-367 
Table I Risk Factors of HIV-Associated Neurocognitive Disorders

\begin{tabular}{|c|c|c|}
\hline Category & Sub-Category & Risk Factors \\
\hline \multirow[t]{7}{*}{ Biological } & Viral-related & $\begin{array}{l}\text { Low nadir CD4 } \\
\text { Duration of infection } \\
\text { Detectable CNS viral load } \\
\text { Detectable plasma viral load } \\
\text { HIV-I Subtype } \\
\text { HII }\end{array}$ \\
\hline & ART-related factors & $\begin{array}{l}\text { Late commencement of ART } \\
\text { ART side effects (metabolic, } \\
\text { neurotoxicity) })^{118} \\
\text { Low CPE scores }^{94} \\
\text { Low ME scores } \\
\text { Sub-optimal ART adherence } \\
\text { ART resistance } \\
\text { 110 }\end{array}$ \\
\hline & Co-morbidities & $\begin{array}{l}\text { CVD (hypertension, diabetes, obesity, } \\
\text { hypercholesteremia, stroke) } \\
\text { Viral co-infection (HCV, HTLV, HIV- } \\
\text { 2) }{ }^{110} \\
\text { Psychiatric disorders (anxiety } \\
\text { disorders, depression, bipolar } \\
\text { disorder) } \\
\text { Sleep disorders (Insomnia, obstructive } \\
\text { sleep apnea) } \\
\text { Epilepsy and seizure disorders } \\
\text { Thrombocytopenia }\end{array}$ \\
\hline & Age & Age $>50$ years $^{122}$ \\
\hline & $\begin{array}{l}\text { CNS Opportunistic } \\
\text { infections }\end{array}$ & $\begin{array}{l}\text { Toxoplasmosis, cryptococcal } \\
\text { meningitis, CNS tuberculosis, CMV }{ }^{123}\end{array}$ \\
\hline & Genetics & $\begin{array}{l}\text { APOE } \varepsilon 4 \text { gene }{ }^{124} \\
\text { Genes relating to serotonin } \\
\text { (SNPrs4570625, GALMrs674/892) }{ }^{113} \\
\text { Genes relating to dopamine (DSD2, } \\
\text { DRD4, DRD3) } \\
\text { Drug metabolism/transporter gene } \\
(\text { CYP2B6) })^{113}\end{array}$ \\
\hline & Head trauma & Traumatic brain injury ${ }^{110}$ \\
\hline Psychological & $\begin{array}{l}\text { Trauma } \\
\text { Stress } \\
\text { Stigma }\end{array}$ & $\begin{array}{l}\text { Chronic exposure to trauma and } \\
\text { violence }{ }^{1 / 4} \\
\text { Early life stress, perceived stress, } \\
\text { PTSD }{ }^{125,126} \\
\text { Experienced, anticipated and } \\
\text { internalized stigma }^{127}\end{array}$ \\
\hline $\begin{array}{l}\text { Social/ } \\
\text { Lifestyle }\end{array}$ & $\begin{array}{l}\text { Employment } \\
\text { Education } \\
\text { Isolation } \\
\text { Poverty } \\
\text { Healthcare access } \\
\text { Exercise }\end{array}$ & $\begin{array}{l}\text { Low paying employment, Low skilled } \\
\text { employment, Unemployment }{ }^{128} \\
\text { Low literacy levels } \\
\text { Living alone } \\
\text { Limited resources and impoverished } \\
\text { communities }^{114} \\
\text { Poor healthcare access }{ }^{113} \\
\text { Low physical activity and vigorous } \\
\text { physical activity }\end{array}$ \\
\hline
\end{tabular}

(Continued)
Table I (Continued).

\begin{tabular}{|l|l|l|}
\hline Category & Sub-Category & Risk Factors \\
\hline & Nutrition & $\begin{array}{l}\text { Poor dietary diversity and vitamin } \\
\text { deficiencies }^{131} \\
\text { Methamphetamine, Alcohol abuse }\end{array}$ \\
\hline
\end{tabular}

Abbreviations: ART, antiretroviral therapy; CPE, central penetrating effectiveness; ME, monocyte efficacy; CVD, cardiovascular disease; HCV, Hepatitis C virus; HTLV, Human T- lymphotropic virus; CMV, cytomegalovirus; PTSD, post-traumatic stress disorder.

Synaptic dendritic networks in the brain undergo continuous remodeling, a process termed neuroplasticity. These changes include increased dendritic branching, augmentation of axonal collaterals, generation of new synaptic connections and activity dependent modification of existing synapses. ${ }^{8}$ The early dementing process in HIV patients has been shown to be associated with synaptic degeneration rather than substantial neuronal apoptosis. ${ }^{9}$ Inhibition of synaptic degeneration therefore provides an attractive therapeutic target to prevent HAND pathogenesis. ${ }^{9}$ Mediators of this CNS plasticity include brain-derived neurotrophic factor (BDNF), insulin-like growth factor (IGF-1), acidic fibroblast growth factor (aFGF), macrophage inflammatory protein-2 (MIP2), stromal derived factor 1 alpha (SDF-1 $\alpha$ ) and leptin. ${ }^{8,9}$ BDNF is the most potent member of the neurotrophin family. ${ }^{10}$

\section{Role of BDNF in HAND Neuropathogenesis}

BDNF is present in tissues both outside and inside the nervous system. It is found in platelets, plasma and serum. It is abundant in various brain regions including the hippocampus, prefrontal cortex and amygdala. ${ }^{10}$ BDNF is synthesized as a larger precursor protein known as preproBDNF that is cleaved into proBDNF which can then be further cleaved by plasma or metalloproteases into mature BDNF. mBDNF and proBDNF elicit opposite effects through the activation of two distinct receptors, Trk and p75NTR, respectively. ${ }^{11}$ Reduced BDNF levels have been associated with numerous structural and functional deficits including loss of cortical and hippocampal synapses, impairment of spatial learning and memory in both rodents and humans. ${ }^{11}$

BDNF plays a critical role in learning and memory. Increased BDNF levels have been shown to decrease the odds of developing HAND. Abassi et al in a Ugandan population, showed lower CSF BDNF levels in HIV patients with 


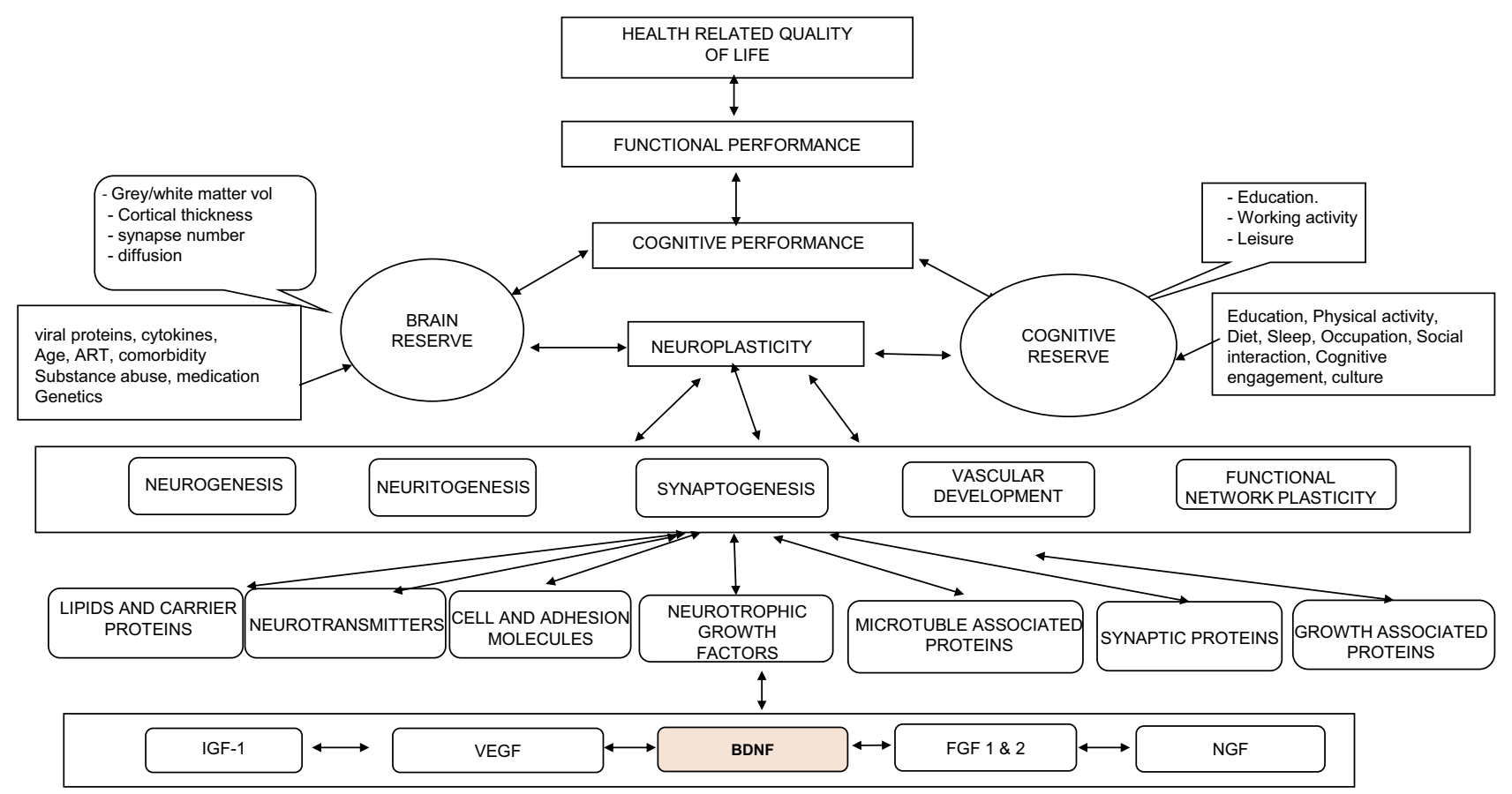

Figure I Biopsychosocial conceptual model of HIV-Associated neurocognitive impairment (neurotrophic pathway). This shows the multicausal factors (behavioral, psychological, sociological and biological) that impacts on cognition vis-à-vis functional impairment and overall health related quality of life. Bidirectional arrows reenforce the complex interactions between factors.

Abbreviations: ART, antiretroviral therapy; BDNF, brain derived neurotrophic factor; VEGF, vascular endothelial growth factor; IGF, insulin-like growth factor; FGF I \& 2, fibroblast growth factor I \& 2; NGF, nerve growth factor.

dementia compared with HIV-positive individuals without dementia. ${ }^{12}$ Falasca et al were able to correlate BDNF levels with different domains of neurocognition. They showed a significant correlation between reduced serum BDNF levels and poor performance on the Grooved Pegboard test for the dominant hand test but no association between BDNF serum levels and attention, executive function and working memory availability. ${ }^{4}$

Processing of proBDNF into mature BDNF has been shown to be reduced in HIV. This contributes to synaptodendritic injury and synaptic dysfunction seen in HAND. Studies have shown that proBDNF levels in HAND subjects were higher than those in HIV-negative as well as HIVpositive subjects without dementia. The reduction in BDNF serum levels in HAND could also be attributed to thrombocytopenia which is quite common in people living with HIV. ${ }^{13}$ Likewise, genetic factors such as rs6265 polymorphism in the BDNF gene could lower BDNF levels observed in HIV-1 positive individuals. This polymorphism results in a Val-Met amino substitution at codon 66 (Val66Met). ${ }^{14}$

Increased TNF- $\alpha$ expression reduces BDNF expression in HIV. TNF- $\alpha$ reduces the anterograde transport of BDNF while IL- $1 \beta$ released in response to gp 120 or tat, decreases the retrograde transport of BDNF. ${ }^{6}$ Preventing disruption in the bidirectional axonal and dendritic transport of BDNF by viral proteins has become a possible therapeutic approach to HAND. ${ }^{15}$

BDNF plays important roles in proliferation, apoptosis and T-cell survival. ${ }^{16}$ BDNF can activate anti-apoptotic genes including Bcl-2 and reduce pro-apoptotic caspase3. ${ }^{17}$ Mature BDNF binds with high affinity to its receptor TrKB. This binding activates TrKB which in turn activates major signaling pathways, one of which is the PLCҮ1-PKC signaling pathway which promotes synaptic plasticity partly by inducing intracellular $\mathrm{Ca}^{2+}$ release. ${ }^{18}$ Other pathways activated include the mutagen activated protein kinase (Ras-MAPK) signaling which promotes neuronal differentiation, the P13 kinase-AK+ signaling which promotes survival and growth of neurons ${ }^{19}$ and the $\operatorname{TrKB} /$ ERK1/2/Ak+ pathway which significantly improves spatial memory. ${ }^{20}$ BDNF has also been shown to activate NF$\mathrm{k} \beta$ which enhances both dendritic spine and excitatory synapse density. ${ }^{18}$ Impairment in spatial learning have been seen in mice lacking in NF-k $\beta$ subunit. By contrast, proBDNF binds with high affinity to $\mathrm{p} 75 \mathrm{NTR} /$ sortilin which in turn activates degeneration of p75NTR positive neurons through a c-Jun N-terminal kinase (JNK)mediated mechanism. ${ }^{21}$ 


\section{BDNF and Viral Proteins}

BDNF reduces degeneration of synapses and axons triggered by viral proteins. These HIV proteins interact with surface receptors on the neurons or activate caspases to effect neuronal damage. The mechanisms by which these viral proteins induce neurotoxicity include production of free radicals, nitric oxide, release of excitotoxins such as glutamate, and through inflammatory cytokines. ${ }^{22}$

Glycoprotein protein 120 (gp120), a viral envelope glycoprotein, evokes neuronal toxicity and reduction of BDNF levels in the dorsal striatum in rodent models of HAND. ${ }^{11}$ This reduction in BDNF levels causes synaptic injury and compromises neuronal function. Neurons exposed to gp120 exhibit lower concentrations of mBDNF and higher levels of proBDNF than controls. ${ }^{3,11}$ In contrast, gp120-upregulated BDNF expression has been shown to contribute to the development of HIV-associated pain through wnt/ $\beta$-catenin signaling in murine derived microglial cell lines. ${ }^{22}$ This supports the evidence that BDNF from sensory neurons plays a critical role in mediating chronic pain. Distal sensory polyneuropathy has been associated with HIV-associated neurocognitive impairment especially with domains containing timed psychomotor tests. ${ }^{23}$ While there has been no evidence correlating elevated neurotoxicity due to increased BDNF levels in the peripheral nervous system with neurotoxicity in the CNS, a few studies have suggested that increased BDNF signaling can be neurotoxic to the brain. ${ }^{24}$ BDNF has been shown to exert dose-dependent opposite effects in the CNS. ${ }^{25}$ In addition to an opposite dose-dependent effect of BDNF, there are also region-specific differences. ${ }^{26}$ Evidence of these divergent effects of BDNF have been seen in the hypothalamus and nucleus accumbens compared to levels in the hippocampus. ${ }^{27}$ It has also been suggested that increased BDNF levels in the CNS microenvironment could increase CCR5 expression which is capable of spreading viral infection. ${ }^{28}$ This highlights the need for more studies to explain the mechanisms responsible for the opposite effects of BDNF expression in different brain regions.

Gp120 shed from HIV binds to chemokine receptors CCR5 and CXCR4. This leads to reduced intracellular processing of proBDNF which appears to occur through a decrease in furin and/or prohormone convertase synthesis/activity. ${ }^{11}$ This increase in proBDNF/mBDNF ratio has been shown to correlate with HIV cognitive impairment in post-mortem samples of HIV-positive individuals. ${ }^{11}$ The increase in CXCR4 expression due to decreased BDNF levels can facilitate entry of HIV into cells and spreading HIV infection. ${ }^{4}$
BDNF, however, has been shown to down regulate the chemokine receptor CXCR4 to which gp120 binds and initiate apoptotic cascade. ${ }^{29}$ This means that increased BDNF levels may reduce gp120 mediated direct neurotoxicity. ${ }^{29}$

Transactivator of transcription (tat) is the first protein to be produced by HIV-infected cell and is associated with BDNF in several ways. It continues to be transcribed in HIVinfected macrophages despite the use of ART and absence of viral replication. ${ }^{30}$ This possibly adds to the reasons why there is still a high prevalence of HAND in the ART era. HIV1-tat coactivator interactions may reduce neurotrophic factor signaling. HIV Tat protein has been found to reduce the expression of BDNF by suppressing BDNF gene expression. Liu et al demonstrated a 50\% decrease in BDNF mRNA levels when neurons were exposed to Tat for $24 \mathrm{hrs} .{ }^{31}$ As a result, HIV tat decreases long-term potentiation (LTP), which is a long-lasting increase in synaptic strength that is critical for learning and memory. HIV Tat achieves this is by decreasing the phosphorylation of the transcription factor cAMP response element binding protein (CREB) thereby downregulating BDNF mRNA levels. ${ }^{32}$

Tat causes dendritic damage which is an important correlate of neurocognitive impairment in HIV and has been associated with specific neurocognitive domains such as learning and verbal fluency. ${ }^{33}$ Conversely, BDNF has been found to support the stability of dendritic spines by increasing the density of dendritic spines possibly through the mechanism of increasing $\mathrm{F}$-actin in spines. ${ }^{31}$ Similar to gp120, tat also decreases the conversion of proBDNF to mBDNF. ${ }^{30}$

Tat increases the activity of NF-k $\beta$ which triggers the activation of immune cells. HIV uses the activation of immune cells as a signal for HIV transcription thereby reactivating the virus from latency, even in the presence of ART. ${ }^{34}$ Altering this process could be beneficial in preventing stealth neurodegeneration. In addition to its functions in the immune system, NF-K $\beta$ regulates neurogenesis and synaptic plasticity. ${ }^{35}$ BDNF has been shown to activate NF-k $\beta$ which enhances both dendritic spine and excitatory synapse density. ${ }^{18}$ Impairment in spatial learning has been seen in mice lacking in the NF-k $\beta$ p50 subunit. ${ }^{36}$ The activation of NF-k $\beta$ by BDNF leads to neuroprotection but chronic activation reduces BDNF and ultimately causes cognitive impairment and neurodegeneration in the dentate gyrus. There is a positive feedback loop between NF-k $\beta$ and BDNF since NF-k $\beta$ also regulates BDNF expression. ${ }^{35}$ 
BDNF has been shown to be neuroprotective by enhancing mitochondrial biogenesis, transport and overall mitochondrial health. ${ }^{24}$ HIV-1 tat and gp120 alter mitochondrial fission, mitochondrial transport and mitophagy in neurons and microglial cells. ${ }^{24}$ Split mitochondria are distributed throughout the soma, dendrites and axons to provide usable energy to synapses for learning, memory and other cognitive functions. This has implicated mitochondrial dysfunction in HAND and illustrates yet another pathway in the role of BDNF in cognitive function. ${ }^{24}$

\section{BDNF and Neurotransmitters in HAND}

HIV impacts negatively on dopaminergic (DA) neurons within the fronto-striato-thalamic system. This causes specific cognitive, motor and behavioral deficits in PLWH. ${ }^{37}$ A decrease in dopamine in the substantia nigra has been associated with poorer cognitive function across specific domains like speed of processing, memory, learning and verbal fluency. ${ }^{38}$ In gp120-treated rats, DA levels were found to be lower than controls in the dorsal striatum and there was loss of nigrostriatal fibers due to intrastriatal gp120. ${ }^{39}$ Recent studies seem to be contradictory showing an increase in DA levels in the CSF of therapynaïve HIV patients. This increase in DA levels was in the asymptomatic stage of infection and could later exert toxic effects in DA neurons due to the oxidative properties of catecholamines. ${ }^{40}$ Increased cytotoxicity of the DA neurons eventually leads to dopamine deficits that may exacerbate the progression of HAND. ${ }^{41}$

Individuals with high dopamine availability have been found to show higher novelty seeking, impulsive, adventure-seeking behavior that can impact sexual behaviors. ${ }^{42}$ This has implications in substance use disorders as exposure to most drugs of abuse raises dopamine levels above the norm associated with the natural reward system. ${ }^{41}$ Early studies have shown association between BDNF and increased dopaminergic neuronal function and survival, as well as tendency to promote the action of drugs of abuse. ${ }^{43}$ Based on BDNF's role in dopamine availability, ${ }^{44}$ the question then arises "Can BDNF levels impact HIV transmission and prevention?". Miguez et al have shown more frequent high-risk sexual behaviors, substance use disorders and sexually transmitted diseases (STDs) in PLWH with low BDNF levels. ${ }^{45}$ There is evidence that activation of D2 receptors also correlates with higher viral loads and production of neurotoxic factors thereby increasing the severity of HAND. $^{46}$

HAND is associated with disrupted glutamate levels. BDNF prevents glutamate mediated neuronal apoptosis by inhibiting caspase 3 activation ${ }^{47}$ and exerts its neuroprotective effect by activating synaptic NMDA receptors, effecting a nuclear calcium genomic response that activates the transcription of inhibin (Activin A). Activin A reduces the neurotoxic NMDA-receptor-mediated calcium influence which shields neurons from mitochondrial dysfunction. ${ }^{48}$

\section{BDNF and Co-Morbid Mental Health Disorders in HAND}

Depression is the most common neuropsychiatric condition in PLWH with a prevalence rate three times that of the general population. ${ }^{49}$ The confounding effect of depression in HAND arises from similar neuropathology which involves the loss of adult neurogenesis in the hippocampus. ${ }^{50} \mathrm{HIV}$ infected individuals however may experience depression despite elevated BDNF levels. ${ }^{4}$ BDNF plays a regulatory role in serotonin systems and, like dopamine, serotonin is also involved in the reward system. ${ }^{51}$

Evidence supporting the BDNF hypothesis of depression in HIV-positive individuals have been lacking as there is little proof relating BDNF signaling to depressive symptoms in HIV. ${ }^{52}$ BDNF is however decreased in the serum of antidepressant-naïve patients. ${ }^{53}$ This shows that while BDNF expression and signaling may not lead to depressive symptoms, it may play a role in the therapeutic effects of antidepressants. ${ }^{54}$ Selective serotonin reuptake inhibitors (SSRIs) increase BDNF levels thereby increasing adult hippocampal neurogenesis. ${ }^{14}$

Lithium and sodium valproate are two commonly used psychotropic drugs which are GSK3B inhibitors. One of the mechanisms by which HIV induces neurotoxicity is through abnormal activation of GSK $3 \beta .^{55}$ GSK3 $\beta$ modulates cellular functions such as gene expression, neurogenesis and synaptic plasticity. It is also a regulator of neuronal and astrocyte cell death in response to neurotoxic stimuli. ${ }^{56}$ This has made GSK 3B an important therapeutic target for HAND. BDNF inhibits GSK3B activity by binding to TrKB to activate PI3K and Akt. ${ }^{57}$ BDNF modulation may therefore be a mechanism by which GSK3B inhibitors like Lithium and sodium valproate ameliorate HIV-1 mediated neurotoxicity. ${ }^{55}$ 


\section{BDNF and Anti-Retrovirals in HAND}

Neurocognitive functioning improves in patients who initiate ART. This shows that ongoing viral replication in the CNS is strongly associated with cognitive impairment. This improvement in neurocognition suggests that alteration of neuronal function prior to cell death may be a likely mechanism accounting for this. ${ }^{58}$ However, some studies have shown dissenting results showing the potential neurotoxicity of ART and worse neuropsychological performance. ${ }^{59}$ It should be noted that some of the reasons for worse neurocognitive outcomes in PLWH on ART could include inhibition of efflux proteins, drug uptake transporters, $A \beta$ and $p$ Tau accumulation, impaired $\mathrm{BBB}$ and immune reconstitution inflammatory syndrome (IRIS) ${ }^{60}$

Long-term administration of nevirapine in mice models showed reduced hippocampal BDNF expression that led to cognitive and behavioral abnormalities while tenofovir had no impact on BDNF expression. ${ }^{61}$ However, there have been conflicting results as regards BDNF levels in ART treated PLWH. Miguez-Burbano et al noticed high levels of circulating BDNF in PLWH in comparison with an HIVnegative control population which may have been the result of increased BDNF synthesis that accompanies the neuronal modelling activated by ART. ${ }^{62}$ AZT-exposed mice showed increased BDNF expression in the hippocampus indicating the initiation of the neuronal survival processes. ${ }^{63}$ More studies are however needed to delineate the mechanisms by which ART impacts on BDNF levels. CCR5 antagonists like Maraviroc have been found to improve the neurocognitive status of $\mathrm{HIV}+$ patients and reduce the expression of several pro-inflammatory factors. ${ }^{64}$ One of the possible mechanisms by which CCR5 antagonists affect neurocognitive benefit could be as a result of increased BDNF expression. ${ }^{65}$

Neurological and psychiatric complications of ART are often difficult to distinguish from HIV-induced peripheral neuropathy. BDNF plays a role in the early stages of zalcitabine-induced mechanical allodynia. ${ }^{25}$ The hypothesis is that BDNF-induced signaling pathway is activated by antiretroviral drug delivery, and this in turn may promote JNK activation and apoptosis, which ultimately leads to mechanical hyperalgesia. BDNF also induces caspase-3 activation which causes cell death. One major mechanism of NRTI-induced polyneuropathy is however mitochondrial toxicity. ${ }^{66}$ There may be some differences in the nature of ART-induced mitochondrial toxicity in the PNS and the CNS but PNS mitochondrial toxicity can provide a possible explanation of how ART may alter mitochondrial function in the brain and cause neurocognitive impairment. ${ }^{24}$ Mitochondrial dysfunction has been implicated in HAND by impairing synaptic plasticity through defective mitochondrial fusion and fission. ${ }^{67}$ BDNF has been shown to support mitochondrial health by enhancing mitochondrial biogenesis, transport and metabolism. ${ }^{24}$ Didanosine also has been shown to diminish expression of BDNF. ${ }^{68}$

Cognitive side effects with Efavirenz have been demonstrated in animal studies. ${ }^{69}$ In a cross-sectional cohort study, HIV-positive subjects who discontinued EFV regimens had greater neurocognitive improvement than those on non-EFV regimens. ${ }^{70} \mathrm{EFV}$ users have been shown to have worse speed of information processing, verbal fluency and working memory when compared to Lopinavir/Ritonavir users in HCV seronegative individuals. ${ }^{71}$ The possible mechanisms of Efavirenz-associated neurocognitive impairment include mitochondrial inhibition, reactive oxygen species generation, dendritic injury and release of cytokines. ${ }^{24,72}$ There are no studies correlating Efavirenz neurotoxicity directly with BDNF levels but with increasing evidence showing BDNF mediation of neurotoxic mechanisms interfering with mitochondrial activity and bioenergetics, it is probable that alteration in BDNF may be implicated in Efavirenz associated HAND. Amprenavir and Lopinavir have been shown to disrupt astrocyte and glutamate functions but neither affected the expression of BDNF in astrocytes. ${ }^{73}$

Zidovudine was shown to decrease P3a latency in HIV patients. P3a latency has been shown to correlate with worsening CNS effect in HIV. ${ }^{74}$ The P3a component of event related potential (ERP) is associated with stimulus novelty processing, allocation of attentional processing resources and cognitive processing speed. However, studies done investigating the role of BDNF in cognitive enhancement using this electrophysiological method showed no correlation with P3a latency. ${ }^{75}$

\section{BDNF and the Human Microbiome in HAND}

Impairment of the microbiota-gut-brain axis in HIVpositive patients may also be implicated in HAND. Some gut bacteria can interact with the CNS to modulate neuroplasticity, thereby affecting cognition and memory. ${ }^{76}$ HIV disrupts the gut microbiota through depletion of the gut epithelial layer and CD4+ $\mathrm{T}$ cells in the gut-associated lymphoid tissue (GALT) ${ }^{77}$ Mounting evidence reveals 
that one of the mechanisms by which the gut microbiota effects neurocognitive changes is through the alteration of hippocampal BDNF expression. ${ }^{78}$ For example, BDNF levels is impacted negatively by Clostridium, Dorea and Blautia but positively by Prevotella sp. ${ }^{79}$

Viruses also have potential functions in the human microbiome. Human endogenous retroviruses (HERV) are a family of viruses within our genome that have similar characteristics with exogenous retroviruses. ${ }^{80}$ HERV expression has been proposed to play a role in CNS disorders like HAND, schizophrenia, bipolar disorder and multiple sclerosis. HIV infection activates HERVs such as HERV-K, HERV-H1F and HERV-W. ${ }^{80}$ HERV $-\mathrm{K}$ is overexpressed in human neurons during HIV/AIDS and have been shown to exert neuroprotective effects in-vitro and in mice model by upregulating BDNF expression as one of its mechanism of action. This regulation of BNDF expression by HERV-K suggests a potential mechanism of reducing neuronal injury. ${ }^{81}$

\section{Potential Role of BDNF in Therapeutic Management of HAND} BDNF has a potential of being more than just an endogenous neuroprotective agent but also a therapeutic immune agent. In addition, BDNF and its modifiers could be helpful in preventative interventions concerning PLWH given the association of neurocognitive impairment with risk-taking behavior. $^{82}$ An ideal drug used in HAND management should be able to penetrate the BBB, be efficacious, affordable, non-invasive and have minimal side effects. ${ }^{83}$ Two options are possible: human BDNF or exogenous agents that can increase endogenous BDNF levels.

\section{Human BDNF}

Human BDNF (hBDNF)-based therapies pose several limitations. The evidence on their efficacy is weak, they are invasive injections, hence unsustainable for chronic administration, and are not readily accessible and affordable. ${ }^{26}$ Tong et al shared their success in using lentivirus as a gene delivery vehicle to administer hBDNF into monocyte derived macrophages. Their invitro study showed the potential of hBDNF cells to protect neuronal cells from TNF- $\alpha$ and HIV-1 Tat mediated neurotoxicity. ${ }^{26}$ There are however no clinical efficacy studies. While there is some enthusiasm with gene therapy, affordability of these therapies is a concern especially in the local resource-constrained neuroAIDS context where
$70 \%$ of all people living with HIV are in Sub-Saharan Africa. ${ }^{1}$

\section{Intranasal Insulin Therapy}

Intranasal insulin therapy in preclinical studies using Eco HIV-infected mice have been shown to improve cognition through upregulation of BDNF levels. ${ }^{84}$ A clinical trial is presently being done by Sacktor et al to assess safety and efficacy in HAND. ${ }^{85}$

\section{Adjunctive Medication}

Adjunctive medication that elevate BDNF levels may also find application in HAND management. Memantine is an uncompetitive antagonist of glutamate NMDA receptors used in the treatment of Alzheimer's disease. It upregulates BDNF expression and has been shown to prevent dopamine deficits in SIV-infected macaques. ${ }^{86}$ Low dose memantine shows some potential in HAND management and treatment of substance use disorders in PLWH. ${ }^{87}$ Antidepressants like the SSRIs (eg, paroxetine) increase BDNF synthesis which causes an increase in hippocampal neural precursor proliferation. ${ }^{88}$ Letendre et al had earlier shown that SSRIs may reduce HIV replication in the CNS and improve neurocognitive performance. This has led to the term "antiviral SSRI". ${ }^{89}$ However, there have been no double-blind randomized controlled clinical trials using SSRIs in HAND therapeutic management.

GSK3B inhibitors-lithium and sodium valproate have also shown some neuroprotective effect through increased synthesis of BDNF in the cerebral cortex. ${ }^{90}$ Statins have immunomodulatory effects and can stimulate BDNF expression. They may be useful in the prevention and management of HAND especially in combating the metabolic effects of HIV and ART. ${ }^{91}$ The increasing age of PLWH creates a higher risk of diabetes and heart disease which can compromise brain function. ${ }^{38}$ Treatment of these co-morbid conditions can possibly increase BDNF levels and improve cognitive deficits. ${ }^{92}$

\section{Anti-Retroviral Therapy (ART)}

ART still remains the most effective HAND management strategy. ${ }^{93}$ Research on the impact of ART on BDNF levels may help efforts to personalizing the ART regimen. Personalized ART regimen has been suggested since benefits of ART in HAND differ between individuals, type of ART, CPE of regimen, time of infection, co-morbidity and as well the neurocognitive domain most affected. ${ }^{94}$ 


\section{Potential Role of BDNF in Non-Pharmacological Approach to HAND Management}

The use of adjuvant medications has the downside of increasing the pill burden and dosing frequency of HIV therapy which can ultimately impact adherence in PLWH already experiencing neurocognitive deficits. ${ }^{95}$ More convenient and safer methods of increasing BDNF have therefore been proffered as the present methods of direct delivery through recombinant protein or viral factors are also cumbersome and invasive. ${ }^{50}$

\section{Transcranial Direct Current Stimulation}

Transcranial Direct Current Stimulation (tDCS) has been proffered as one of the potential non-pharmacologic approaches to HAND. tDCS of motor cortex neurons in HIV patients have shown benefit in increasing BDNF levels and enhancing neurocognitive performance. ${ }^{96}$ This technique holds some promise in HAND management as it is affordable and relatively safe. Ownby et al are presently conducting a clinical trial to investigate the effectiveness of computer-based cognitive training and tDCS in enhancing neurocognitive performance in older HIV-positive individuals. ${ }^{97}$

\section{Diet}

A few dietary phytochemicals have been proposed to induce hippocampal BDNF expression. Flavonoids like hesperetin, quercetin, naringenin and luteolin have been shown to improve cognition by promoting neurogenesis and synaptic plasticity through serum BDNF changes. Dietary flavonoids occur naturally in fruits, vegetables, chocolates and beverages like wine and tea, ${ }^{98}$ which makes them easily accessible and cost-effective. There is however no clinical evidence showing their efficacy in HIV-associated neurocognitive disorders. Luteolin has however shown significant anti-HIV activity in reporter cells and primary lymphocytes at the Tat-long terminal repeat transactivation level. ${ }^{99}$ Such flavonoids with BDNF expression activity as well as antiHIV property hold some promise as adjunctive treatment with ART for the management of HAND. BDNF has also been found to mediate the neuroprotective actions of catechins (found in tea leaves, apple, grapes and red wine), rosmarinic acid (found in culinary herbs) and curcumin (found in turmeric). ${ }^{21}$

Probiotics, prebiotics (dietary fibers and oligosaccharides), omega-3-fatty acids and fermented foods (eg, yoghurt) have been shown to increase BDNF levels and improve cognition through their modulation of the gut microbiota. This have led to the term "psychobiotics". ${ }^{100}$ Probiotics like lactobacillus, Bifidobacterium, Prevotella, Faecalibacterium prausnitzii have shown potential in preventing or slowing neurodegenerative processes. ${ }^{76,79}$ However, there is little evidence for the efficacy of probiotics in HAND. Ceccarelli et al showed improvement of neuropsychological performance in an HIV positive 56-year-old Caucasian male after 6 months of probiotic supplementation. ${ }^{76}$ The neurocognitive effect of probiotics in association with ART therapy needs to be explored with larger samples of patients to provide conclusive evidence of their role in HAND.

\section{Physical Exercise}

Incorporating physical exercise to drug therapy may be another approach to increasing BDNF levels and neurocognition in HIV patients. ${ }^{50}$ Exercise has been found in a HIVgp120 transgenic mouse model to restore BDNF levels through cdk5 regulation which consequently normalizes dendritic branching. ${ }^{88}$ Aerobic exercise such as walking and stretching enhance neural plasticity by upregulation of BDNF. ${ }^{101}$ However, no studies have explored the effect of exercise and BDNF levels in HIV-positive individuals.

\section{Sleep}

The prevalence of sleep disturbances in PLWH is estimated at $58 \% .{ }^{102}$ The quality and duration of sleep can affect cognition in HIV-positive patients. This has been confirmed through objective and subjective measures of sleep. ${ }^{103}$ Sleep improves synaptic plasticity, memory and learning hence reduced cognition has been correlated with decreased peripheral BDNF levels. ${ }^{104}$ Non-pharmacologic interventions like cognitive behavioral therapy to help with sleep have been suggested but to date, the evidence for improving cognitive functioning in PLWH is not strong. ${ }^{105}$

\section{Building Cognitive Reserve}

In the presence of brain pathology, some individuals function better than others, showing better adaptation of cognitive networks; this phenomenon has been termed cognitive reserve (CR). ${ }^{106}$ The concept of "reserve" has been richly explored in Alzheimer's disease. In AD pathology, high BDNF expression has shown to enhance CR. ${ }^{107}$ There is need to translate this concept into HAND research and explore the impact of social interactions, environmental enrichment through education and physical activity on BDNF and cognition in PLWH. Knowing that learning and novelty stimulate neural plasticity, ${ }^{108}$ there is an opportunity 
to also explore interventional cognitive neurorehabilitation tools such as complex gameplay in PLWH and how they modulate BDNF levels.

\section{Research Gaps and Future Direction}

There is a need to further investigate the dual nature of BDNF effects so as to be able to modulate its levels for neuroprotection without detrimental effects as seen in nociceptive processes. The methodology of BDNF assessment will need to be strengthened such that the proBDNF and mBDNF isoforms are analysed and reported separately. This is necessary since the proBDNF isoform is associated with neurotoxicity and the mBDNF form with neuroprotection. ${ }^{109}$

There is a gap in literature on the potential impact of BDNF on risk-taking behavior and decision making. Future research will need to examine the impact on BDNF levels on cognitive domains of executive function which is critical for adherence to ART, safe sex and impulse control as regards substance use. ${ }^{110}$

Most studies on the effect of BDNF on neurocognition in HIV-positive individuals have been conducted in high-income countries with a prevalence of HIV-1 Subtype B. It is therefore important to study the role of BDNF within regions like sub-Saharan Africa which is the epicenter of the HIV epidemic. This recommendation draws from the knowledge that the HIV-1 sub-type $\mathrm{C}$ virus predominates in this region and variation in the neurovirulence and synaptodendritic alteration effect of HIV-1 subtypes have been established. ${ }^{111,112}$ In addition, the association of low cognitive reserve with neurocognitive impairment in $\mathrm{HIV},{ }^{113}$ makes it imperative to study the impact of confounding risk factors which are abundant in sub-Saharan Africa and their impact on BDNF and neurocognition. These factors include low literacy levels, limited access to healthcare, chronic exposure to trauma, poor dietary diversity, higher rates of treatment failure and late-onset of treatment. ${ }^{114}$

There is also low clinical knowledge of the acute and chronic impact of antiretroviral therapy on BDNF expression. Results of such studies coupled with BDNFmodulatory adjunctive medication for co-morbid disorders and as well BDNF-inspired non-pharmacological interventions can feed into designing personalized treatment for people living with HIV with neurocognitive impairment.

\section{Conclusion}

HAND impacts on the everyday functionality of PLWH despite treatment with ART. There is a critical need to develop treatment strategies to tackle the neurotoxic mechanisms in HAND and the resulting clinical deficits, especially in light of the high prevalence of HIV and increased survival rates accorded by ART. The human brain is complex. As shown in Figure 1, it will be simplistic to assume a "one-neuromarker-fits-all" approach to HAND management. A holistic approach of using neuromarkers, ART, adjunctive therapy for co-morbid disorders tailored to optimize neurocognitive outcomes, supportive cognitive and behavioral therapies and genomics will be required to effectively target neurocognition and reduce the prevalence of HAND in the ART era. However, integrating antiretroviral therapy, adjunctive therapy and nonpharmacological approaches to enhance BDNF expression vis-à-vis neurocognition may be a possible beneficial strategy in tacking HAND.

There is a need to identify biomarkers for HAND. BDNF could be one such biomarker that has a modest yet growing body of evidence in in-vitro, animal and human studies. Moreover, there is the exciting potential to access the BDNF system through non-invasive, non-pharmacological and possibly cost-effective ways that are necessary in local contexts of high disease burden and resource constraints.

\section{Disclosure}

The authors report no conflicts of interest in this work.

\section{References}

1. UNAIDS. UNAIDS data; 2018. Available from: https://www.unaids. org/sites/default/files/media_asset/unaids-data-2018_en.pdf. Accessed June 18, 2019.

2. Rumbaugh JA, Tyor W. HIV-associated neurocognitive disorders: five new things. Neurol Clin Pract. 2015;5(3):224-231. doi:10.1212/ CPJ.0000000000000117

3. Avdoshina V, Bachis A, Mocchetti I. Synaptic dysfunction in human immunodeficiency virus type-1-positive subjects: inflammation or impaired neuronal plasticity? J Intern Med. 2013;273(5):454-465. doi:10.1111/joim.12050

4. Falasca K, Reale M, Ucciferri C, et al. Cytokines, hepatic fibrosis, and antiretroviral therapy role in neurocognitive disorders HIV related. AIDS Res Hum Retroviruses. 2017;33(3):246-253. doi:10.1089/ aid.2016.0138

5. Vally Z. HIV-associated neurocognitive disorders. South African J Psychiatry. 2011;17(4):4. doi:10.4102/sajpsychiatry.v17i4.294

6. Wenzel ED, Avdoshina V, Mocchetti I. HIV-associated neurodegeneration: exploitation of the neuronal cytoskeleton. J Neurovirol. 2019;25 (3):301-312. doi:10.1007/s13365-019-00737-y

7. Kays JL, Psy D, Hurley RA, Taber KH, Ph D. The dynamic brain: neuroplasticity and mental health. $J$ Neuropsychiatry Clin Neurosci. 2012;24(2):118-124. doi:10.1176/appi.neuropsych.12050109

8. Ellis RJ, Calero P, Stockin MD. HIV infection and the central nervous system: a primer. Neuropsychol Rev. 2009;19(2):144-151. doi:10.10 07/s11065-009-9094-1 
9. Joska JA, Hoare J, Stein DJ, Flisher AJ. The neurobiology of HIV dementia: implications for practice in South Africa. Afr J Psychiatry. 2011;14(1):17-22. doi:10.4314/ajpsy.v14i1.65463

10. Schmitt K, Holsboer-Trachsler E, Eckert A. BDNF in sleep, insomnia, and sleep deprivation. Ann Med. 2016;48(1-2):42-51. doi: 10.3109/07853890.2015.1131327

11. Bachis A, Avdoshina V, Zecca L, Parsadanian M, Mocchetti I. Human immunodeficiency virus type 1 alters brain-derived neurotrophic factor processing in neurons. $J$ Neurosci. 2012;32 (28):9477-9484. doi:10.1523/JNEUROSCI.0865-12.2012

12. Abassi M, Morawski BM, Nakigozi G, et al. Cerebrospinal fluid biomarkers and HIV-associated neurocognitive disorders in HIV-infected individuals in Rakai, Uganda. $J$ Neurovirol. 2017;23(3):369-375. doi:10.1007/s13365-016-0505-9

13. Nascimento FG, Tanaka PY. Thrombocytopenia in HIV-infected patients. Indian J Hematol Blood Transfus. 2012;28(2):109-111. doi:10.1007/s12288-011-0124-9

14. Avdoshina V, Garzino-Demo A, Bachis A, et al. HIV-1 decreases the levels of neurotrophins in human lymphocytes. AIDS. 2011;25 (8):1126-1128. doi:10.1097/QAD.0b013e32834671b3

15. Adachi N, Numakawa T, Richards M, Nakajima S, Kunugi H. New insight in expression, transport, and secretion of brain-derived neurotrophic factor: implications in brain-related diseases. World J Biol Chem. 2014;5(4):409-428. doi:10.4331/ wjbc.v5.i4.409

16. Míguez-Burbano MJ, Espinoza L, Bueno D, et al. Beyond the brain: the role of brain-derived neurotrophic factor in viroimmune responses to antiretroviral therapy among people living with HIV with and without alcohol use. J Int Assoc Provid AIDS Care. 2014;13(5):454-460. doi:10.1177/232595741453 5253

17. Kovalevich J, Langford D. Neuronal toxicity in HIV CNS disease. Future Virol. 2012;7(7):687-698. doi:10.2217/fvl.12.57

18. Finley J. Facilitation of hippocampal long-term potentiation and reactivation of latent HIV-1 via AMPK activation: common mechanism of action linking learning, memory, and the potential eradication of HIV-1. Med Hypotheses. 2018;116(April):61-73. doi:10.1016/j.mehy.2018.04.018

19. Miguez-burbano M. HIV, alcohol use disorders and pain: new findings to address the problem. J HIV Retro Virus. 2017;3 (1):1-6. doi:10.21767/2471-9676.100029

20. Wu Y, Luo X, Liu X, et al. Intraperitoneal administration of a novel TAT-BDNF peptide ameliorates cognitive impairments via modulating multiple pathways in two Alzheimer's rodent models. Sci Rep. 2015;5:15032. doi:10.1038/srep15032

21. Nath S, Bachani M, Harshavardhana D, Steiner JP. Catechins protect neurons against mitochondrial toxins and HIV proteins via activation of the BDNF pathway. $J$ Neurovirol. 2012;18 (6):445-455. doi:10.1007/s13365-012-0122-1

22. Wang Y, Liao J, Tang S-J, Shu J, Zhang W. HIV-1 gp120 upregulates brain-derived neurotrophic factor (BDNF) expression in BV2 cells via the Wnt/ $\beta$-Catenin Signaling pathway. $J \mathrm{Mol}$ Neurosci. 2017;62(2):199-208. doi:10.1007/s12031-017-0931-z

23. Fellows RP, Byrd DA, Elliott K, et al. Distal sensory polyneuropathy is associated with neuropsychological test performance among persons with HIV. J Int Neuropsychol Soc. 2012;18 (5):898-907. doi:10.1017/S1355617712000707

24. Fields JA, Ellis RJ. HIV in the CART Era and the Mitochondrial: ImmuneInterface in the CNS. Vol. 145. 1st ed. Elsevier Inc.; 2019 doi:10.1016/bs.irn.2019.04.003

25. Sanna MD, Ghelardini C, Galeotti N. Neuropharmacology blockade of the spinal BDNF-activated JNK pathway prevents the development of antiretroviral-induced neuropathic pain. Neuropharmacology. 2016;105:543-552. doi:10.1016/j.neuro pharm.2016.02.016
26. Tong J, Buch S, Yao H, et al. Monocytes-derived macrophages mediated stable expression of human brain-derived neurotrophic factor, a novel therapeutic strategy for NeuroAIDS. PLoS One. 2014;9(2):1-12. doi:10.1371/journal.pone.0082030

27. Lakshminarasimhan $\mathrm{H}$, Chattarji S. Stress leads to contrasting effects on the levels of brain derived neurotrophic factor in the hippocampus and amygdala. PLoS One. 2012;7(1):e30481. doi:10.1371/journal.pone.0030481

28. Harrold SM, Dragic JM, Brown SL, Achim CL. Neurotrophic factor regulation of human immunodeficiency virus type 1 replication in human blood-derived macrophages through modulation of coreceptor expression. Adv Exp Med Biol. 2001;493:41-47. doi:10.1007/0-306-47611-8_5

29. Mocchetti I, Bachis A, Avdoshina V. Neurotoxicity of human immunodeficiency virus-1: viral proteins and axonal transport. Neurotox Res. 2012;21(1):79-89. doi:10.1007/s12640-011-9279-2

30. Carvallo L, Lopez L, Fajardo JE, Jaureguiberry-Bravo M, Fiser A, Berman JW. HIV-Tat regulates macrophage gene expression in the context of neuroAIDS. PLoS One. 2017;12(6): e0179882-e0179882. doi:10.1371/journal.pone.0179882

31. Liu Y, Zhou D, Feng J, et al. HIV-1 protein Tat(1-72) impairs neuronal dendrites via activation of PP1 and regulation of the CREB/BDNF pathway. Virol Sin. 2018;33(3):261-269. doi:10.1007/s12250-018-0031-4

32. Santerre M, Bagashev A, Gorecki L, et al. HIV-1 tat protein promotes neuronal dysregulation by inhibiting E2F transcription factor 3. J Biol Chem. 2018;3(6):1-29. doi:10.1074/jbc.RA118.003744

33. Masliah E, Heaton RK, Marcotte TD, et al. Dendritic injury is a pathological substrate for human immunodeficiency virus-related cognitive disorders. Ann Neurol. 1997;42 (6):963-972. doi:10.1002/ana.410420618

34. Ambrosius B, Gold R, Chan A, Faissner S. Antineuroinflammatory drugs in HIV-associated neurocognitive disorders as potential therapy. Neurol Neuroimmunol Neuroinflamm. 2019;6(3):e551. doi:10.1212/NXI.0000000000000551

35. Caviedes A, Lafourcade C, Soto C, Wyneken U. BDNF/NF- $\kappa B$ signaling in the neurobiology of depression. Curr Pharm Des. 2017;23(21):3154-3163. doi:10.2174/138161282366617011114 1915

36. Oikawa K, Odero GL, Platt E, et al. NF-кB p50 subunit knockout impairs late LTP and alters long term memory in the mouse hippocampus. BMC Neurosci. 2012;13(1):45. doi:10.1186/14712202-13-45

37. Levine AJ, Sinsheimer JS, Bilder R, Shapshak P, Singer EJ. Functional polymorphisms in dopamine-related genes: effect on neurocognitive functioning in $\mathrm{HIV}+$ adults. $J$ Clin Exp Neuropsychol. 2012;34(1):78-91. doi:10.1080/13803395.2011. 623118

38. Cody SL, Vance DE. Neurobiology of disease the neurobiology of HIV and its impact on cognitive reserve: a review of cognitive interventions for an aging population. Neurobiol Dis. 2016;92 (PartB):144-156. doi:10.1016/j.nbd.2016.01.011

39. Mocchetti I, Nosheny RL, Tanda G, Ren KE, Meyer EM. Brainderived neurotrophic factor prevents human immunodeficiency virus type 1 protein gp120 neurotoxicity in the rat nigrostriatal system. Ann N Y Acad Sci. 2007;1122(1):144-154. doi:10.1196/ annals. 1403.010

40. Scheller C, Arendt G, Nolting T, et al. Increased dopaminergic neurotransmission in therapy-naïve asymptomatic HIV patients is not associated with adaptive changes at the dopaminergic synapses. J Neural Transm. 2010;117(6):699-705. doi:10.1007/ s00702-010-0415-6

41. Purohit V, Rapaka R, Shurtleff D. Drugs of abuse, dopamine, and HIV-associated neurocognitive disorders/HIV-associated dementia. Mol Neurobiol. 2011;44(1):102-110. doi:10.1007/s12 035-011-8195-z 
42. Horn A, Scheller CS, Burger PR. The dopamine-related polymorphisms BDNF, COMT, DRD2, DRD3, and DRD4 are not linked with changes in CSF dopamine levels and frequency of HIV infection. J Neural Transm. 2016;124(4):501-509. doi:10. 1007/s00702-016-1659-6

43. Horger BA, Iyasere CA, Berhow MT, Messer CJ, Nestler EJ, Taylor JR. Enhancement of locomotor activity and conditioned reward to cocaine by brain-derived neurotrophic factor. $J$ Neurosci. 1999;19(10):4110LP- 4122. doi:10.1523/JNEURO SCI.19-10-04110.1999

44. Mocchetti I, Bachis A, Nosheny RL, Tanda G. Brain-derived neurotrophic factor expression in the substantia nigra does not change after lesions of dopaminergic neurons. Neurotox Res. 2007;12(2):135-143. doi:10.1007/BF03033922

45. Míguez MJ, Espinoza LA, Perez C, Kahler C. Brain derived neurotrophic factor: from neurobiology to sexual risk prevention. J AIDS Clin Res. 2016;7:10. doi:10.4172/21556113.1000620

46. Del Guerra FB, Fonseca JLI, Figueiredo VM, Ziff EB, Konkiewitz EC. Human immunodeficiency virus-associated depression: contributions of immuno-inflammatory, monoaminergic, neurodegenerative, and neurotrophic pathways. $J$ Neurovirol. 2013;19(4):314-327. doi:10.1007/s13365-013-0177-7

47. Bachis A, Major EO, Mocchetti I. Brain-Derived neurotrophic factor inhibits human immunodeficiency virus-1/gp120-mediated cerebellar granule cell death by preventing gp120 internalization. J Neurosci. 2003;23(13):5715LP- 5722. doi:10.1523/JNEUROSCI. 23-13-05715.2003

48. Lau D, Bengtson CP, Buchthal B, Bading H. BDNF reduces toxic extrasynaptic NMDA receptor signaling via synaptic NMDA receptors and nuclear-calcium-induced transcription of inhba/activin A. Cell Rep. 2015;12(8):1353-1366. doi:10.1016/j.celrep. 2015.07.038

49. Rubin LH, Maki PM, Rubin LH. HIV, depression, and cognitive impairment in the era of effective antiretroviral therapy. Curr HIV/AIDS Rep. 2019;16(1):82-95. doi:10.1007/s11904-01900421-0

50. Mocchetti I, Bachis A, Campbell LA. Implementing neuronal plasticity in NeuroAIDS: the experience of brain-derived neurotrophic factor and other neurotrophic factors. J Neuroimmune Pharmacol. 2014;9(2):80-91. doi:10.1007/s11481-013-9488-y

51. Mb MJ, Richardson E, Vargas M, et al. Mentholated cigarettes are related with abnormal brain-derived neurotrophic factor levels among smokers living with HIV. Alcohol Drug Depend. 2014;2:5. doi:10.4172/2329-6488.10001

52. Avdoshina V, Mocchetti I, Liu C, et al. Single-nucleotide polymorphisms in TrkB and risk for depression: findings from the women's interagency HIV study. J Acquir Immune Defic Syndr. 2013;64(2):138-141. doi:10.1097/QAI.0b013e3182a468e9

53. Nosheny RL, Ahmed F, Yakovlev A, et al. Brain-derived neurotrophic factor prevents the nigrostriatal degeneration induced by human immunodeficiency virus-1 glycoprotein 120 in vivo. Eur $J$ Neurosci. 2007;25(8):2275-2284. doi:10.1111/j.1460-9568.20 07.05506.x

54. Duclot F, Kabbaj M. Epigenetic mechanisms underlying the role of brain-derived neurotrophic factor in depression and response to antidepressants. J Exp Biol. 2015;218(Pt 1):21-31. doi:10.1242/ jeb. 107086

55. Masvekar RR, El-Hage N, Hauser KF, Knapp PE. Morphine enhances HIV-1SF162-mediated neuron death and delays recovery of injured neurites. PLoS One. 2014;9(6):e100196-e100196. doi:10.1371/journal.pone. 0100196

56. Khan I, Tantray MA, Alam MS, Hamid H. Natural and synthetic bioactive inhibitors of glycogen synthase kinase. Eur $\mathrm{J} \mathrm{Med}$ Chem. 2017;125:464-477. doi:10.1016/j.ejmech.2016.09.058
57. Xu L-Z, Xu D-F, Han Y, et al. BDNF-GSK-3 $\beta-\beta-$-Catenin pathway in the $\mathrm{mPFC}$ is involved in antidepressant-like effects of morinda officinalis oligosaccharides in rats. Int $J$ Neuropsychopharmacol. 2016;20(1):83-93. doi:10.1093/ijnp/pyw088

58. Robertson KR, Su Z, Margolis DM, et al. Neurocognitive effects of treatment interruption in stable HIV-positive patients in an observational cohort. Neurology. 2010;74(16):1260LP-1266. doi:10.1212/WNL.0b013e3181d9ed09

59. Brier MR, Wu Q, Tanenbaum AB, Westerhaus ET, Kharasch ED, Ances BM. Effect of HAART on brain organization and function in HIV-negative subjects. J Neuroimmune Pharmacol. 2015;10 (4):517-521. doi:10.1007/s11481-015-9634-9

60. Shah A, Gangwani MR, Chaudhari NS, Glazyrin A, Bhat HK, Kumar A. Neurotoxicity in the post-HAART era: caution for the antiretroviral therapeutics. Neurotox Res. 2016;30(4):677-697. doi:10.1007/s12640-016-9646-0

61. Zulu SS, Simola N, Mabandla MV, Daniels WMU. Effect of long-term administration of antiretroviral drugs (Tenofovir and Nevirapine) on neuroinflammation and neuroplasticity in mouse hippocampi. $J$ Chem Neuroanat. 2018;94(October):86-92. doi:10.1016/j.jchemneu.2018.10.003

62. Míguez-Burbano MJ, Espinoza L, Whitehead NE, et al. Brain derived neurotrophic factor and cognitive status: the delicate balance among people living with HIV, with and without alcohol abuse. Curr HIV Res. 2014;12(4):254-264.

63. Calamandrei G, Valanzano A, Puopolo M, Aloe L. Developmental exposure to the antiretroviral drug zidovudine increases brain levels of brain-derived neurotrophic factor in mice. Neurosci Lett. 2002;333(2):111-114. doi:10.1016/S0304-3940(02)01023-6

64. Kim S, Hahn YK, Podhaizer EM, et al. A central role for glial CCR5 in directing the neuropathological interactions of HIV-1 Tat and opiates. $J$ Neuroinflammation. 2018;15(1):285. doi:10.1186/s12974-018-1320-4

65. Victoria ECG, de Brito Toscano EC, de Sousa Cardoso AC, et al. Knockdown of $\mathrm{C}-\mathrm{C}$ chemokine receptor 5 (CCR5) is protective against cerebral ischemia and reperfusion injury. Curr Neurovasc Res. 2017;14(2):125-131. doi:10.2174/15672026146666170313113 056

66. Dalakas MC. Peripheral neuropathy and antiretroviral drugs. $J$ Peripher Nerv Syst. 2001;6(1):14-20. doi:10.1046/j.15298027.2001.006001014.x

67. Chen F, Ardalan M, Elfving B, Wegener G, Madsen TM, Nyengaard JR. Mitochondria are critical for BDNF-mediated synaptic and vascular plasticity of hippocampus following repeated electroconvulsive seizures. Int $J$ Neuropsychopharmacol. 2017;21 (3):291-304. doi:10.1093/ijnp/pyx115

68. Zhu Y, Antony JM, Martinez JA, et al. Didanosine causes sensory neuropathy in an HIV/AIDS animal model: impaired mitochondrial and neurotrophic factor gene expression. Brain. 2007;130 (8):2011-2023. doi:10.1093/brain/awm148

69. Romão PRT, Lemos JC, Moreira J, et al. Anti-HIV drugs nevirapine and efavirenz affect anxiety-related behavior and cognitive performance in mice. Neurotox Res. 2011;19(1):73-80. doi:10.1007/s12640-009-9141-y

70. Ciccarelli N, Fabbiani M, Di Giambenedetto S, et al. Efavirenz associated with cognitive disorders in otherwise asymptomatic HIV-infected patients. Neurology. 2011;76(16):1403LP- 1409. doi:10.1212/WNL.0b013e31821670fb

71. Ma Q, Vaida F, Wong J, et al. Long-term efavirenz use is associated with worse neurocognitive functioning in HIV-infected patients. J Neurovirol. 2016;22(2):170-178. doi:10.1007/s13365015-0382-7

72. Apostolova N, Funes HA, Blas-garcia A, et al. Efavirenz and the CNS: what we already know and questions that need to be answered. J Antimicrob Chemother. 2015;70(July):2693-2708. doi:10.1093/jac/dkv183 
73. Vivithanaporn P, Asahchop EL, Acharjee S, Baker GB, Power C. HIV protease inhibitors disrupt astrocytic glutamate transporter function and neurobehavioral performance. AIDS. 2016;30 (4):543-552. doi:10.1097/QAD.0000000000000955

74. Evers S, Husstedt I-W, Luttmann S, Bauer B, Grotemeyer K-H. Event-related potentials in HIV infection: evidence for impact of antiretroviral treatment. Arch Neurol. 1996;53(8):715-716. doi:10.1001/archneur.1996.00550080021005

75. Soltész F, Suckling J, Lawrence P, et al. Identification of BDNF sensitive electrophysiological markers of synaptic activity and their structural correlates in healthy subjects using a genetic approach utilizing the functional BDNF Val66Met polymorphism. PLoS One. 2014;9:4. doi:10.1371/journal.pone.0095558

76. Ceccarelli G, Vassalini P, Scheri GC, et al. Improvement of neuropsychological performances and reduction of immune-activation markers after probiotic supplementation and change of life-style in an HIV positive male: targeting the microbiota to act on gut-brain axis. Infect Dis Trop Med. 2017;3(3):1-5.

77. Li SX, Armstrong A, Neff CP, Shaffer M, Lozupone CA, Palmer BE. Complexities of gut microbiome dysbiosis in the context of HIV infection and antiretroviral therapy. Clin Pharmacol Ther. 2016;99(6):600-611. doi:10.1002/cpt.363

78. Borrelli L, Aceto S, Agnisola C, et al. Probiotic modulation of the microbiota-gut-brain axis and behaviour in zebrafish. Sci Rep. 2016;6:30046. doi:10.1038/srep30046

79. Prykhodko O, Sandberg J, Burleigh S, Björck I, Nilsson A, Fåk Hållenius $\mathrm{F}$. Impact of rye kernel-based evening meal on microbiota composition of young healthy lean volunteers with an emphasis on their hormonal and appetite regulations, and blood levels of brain-derived neurotrophic factor. Front Nutr. 2018;5:45. doi:10.3389/fnut.2018.00045

80. Tove C. Human endogenous retroviruses in neurological diseases. Trends Mol Med. 2018;24(4):379-394. doi:10.1016/j.molmed.2018. 02.007

81. Bhat RK, Rudnick W, Antony JM, et al. Human endogenous retrovirus-K(II) envelope induction protects neurons during HIV/AIDS. PLoS One. 2014;9(7):e97984-e97984. doi:10.1371/ journal.pone. 0097984

82. Forstein Marshall. Depression in the Aging HIV Infected Population - HIV and Aging. HIV and Aging; 2016. Available from: http://hiv-age.org/2016/01/26/depression-in-the-aging-hivinfected-population/. Accessed June 5, 2019.

83. Tang H, Lu D, Pan R, Qin X, Xiong H, Dong J. Curcumin improves spatial memory impairment induced by human immunodeficiency virus type 1 glycoprotein $120 \mathrm{~V} 3$ loop peptide in rats. Life Sci. 2009;85(1-2):1-10. doi:10.1016/j.1fs.2009.03.013

84. Kim B-H, Kelschenbach J, Borjabad A, et al. Intranasal insulin therapy reverses hippocampal dendritic injury and cognitive impairment in a model of HIV-associated neurocognitive disorders in EcoHIV-infected mice. AIDS. 2019;33(6):973-984. doi:10.1097/QAD.0000000000002150

85. John Hopkins University. Intranasal insulin for the treatment of HAND. Available from: https://clinicaltrials.gov/ct2/show/ NCT03081117. NLM identifier: NCT03081117. Accessed July 7, 2019.

86. Meisner F, Scheller C, Kneitz S, et al. Memantine upregulates BDNF and prevents dopamine deficits in SIV-Infected Macaques: a novel pharmacological action of memantine. Neuropsychopharmacology. 2008;33(9):2228-2236. doi:10.1038/sj.npp.1301615

87. Chang SL. Potential therapeutic strategy to treat substance abuse related disorders. J Food Drug Anal. 2013;21(4):S25-S26. doi:10.1016/j.jfda.2013.09.028

88. Lee M-H, Amin ND, Venkatesan A, et al. Impaired neurogenesis and neurite outgrowth in an HIV-gp120 transgenic model is reversed by exercise via BDNF production and Cdk5 regulation. $J$ Neurovirol. 2013;19(5):418-431. doi:10.1007/s13365-013-0194-6
89. Letendre SL, Marquie-Beck J, Ellis RJ, et al. The role of cohort studies in drug development: clinical evidence of antiviral activity of serotonin reuptake inhibitors and HMG-CoA reductase inhibitors in the central nervous system. J Neuroimmune Pharmacol. 2007;2(1):120. doi:10.1007/s11481-006-9054-y

90. Mocchetti I, Bachis A, Masliah E. Review chemokine receptors and neurotrophic factors: potential therapy against AIDS dementia? J Neurosci Res. 2008;255(March2007):243-255. doi:10.1002/jnr

91. Yadav A, Betts MR, Collman RG. Statin modulation of monocyte phenotype and function: implications for HIV-1-associated neurocognitive disorders. $J$ Neurovirol. 2016;22(5):584-596. doi:10.1007/s13365-016-0433-8

92. Jin $\mathrm{H}$, Chen $\mathrm{Y}$, Wang B, et al. Association between brain-derived neurotrophic factor and von Willebrand factor levels in patients with stable coronary artery disease. BMC Cardiovasc Disord. 2018;18(1):23. doi:10.1186/s12872-018-0762-z

93. Braganca M, Palha A. Trastornos neurocognitivos asociados con la infección por el VIH [HIV associated neurocognitive disorders]. Actas Esp Psiquiatr. 2011;39(6):374-383. Spanish.

94. Carvalhal A, Gill MJ, Letendre SL, et al. Central nervous system penetration effectiveness of antiretroviral drugs and neuropsychological impairment in the ontario HIV treatment network cohort study. J Neurovirol. 2016;22(3):349-357. doi:10.1007/s13365-015-0404-5

95. Ettenhofer ML, Foley J, Castellon SA, Hinkin CH. Reciprocal prediction of medication adherence and neurocognition in HIV/ AIDS. Neurology. 2010;74(15):1217-1222. doi:10.1212/WNL.0b 013e3181d8c1ca

96. Periyasamy P, Guo M-L, Buch S. Growth and neurotrophic factors in HIV-associated neurocognitive disorders BT. In: Ikezu T, Gendelman HE, editors. Neuroimmune Pharmacology. Cham: Springer International Publishing; 2017:285-298. doi:10.1007/ 978-3-319-44022-4_18

97. Ownby RL. Cognitive training and transcranial direct current stimulation for cognition in HIV. Available from: https://clinicaltrials.gov/ ct2/show/NCT03440840. NLM identifier: NCT03440840 Accessed August 1, 2019.

98. Neshatdoust S, Saunders C, Castle SM, et al. High-flavonoid intake induces cognitive improvements linked to changes in serum brain-derived neurotrophic factor: two randomised, controlled trials. Nutr Heal Aging. 2016;4(1):81-93. doi:10.3233/ NHA-1615

99. Mehla R, Bivalkar-Mehla S, Chauhan A. A flavonoid, luteolin, cripples HIV-1 by abrogation of tat function. PLoS One. 2011;6 (11):e27915-e27915. doi:10.1371/journal.pone.0027915

100. Dinan TG, Stanton C, Cryan JF. Psychobiotics: a novel class of psychotropic. Biol Psychiatry. 2013;74(10):720-726. doi:10.101 6/j.biopsych.2013.05.001

101. Leckie RL, Oberlin LE, Voss MW, et al. BDNF mediates improvements in executive function following a 1-year exercise intervention. Front Hum Neurosci. 2014;8:985.

102. Oshinaike O, Akinbami A, Ojelabi O, Dada A, Dosunmu A, John Olabode S. Quality of sleep in an HIV population on antiretroviral therapy at an urban tertiary centre in lagos. Nigeria. 2014;2014:1-6. doi:10.1155/2014/298703

103. Mahmood Z, Hammond A, Nunez RA, Irwin MR, Thames AD. Effects of sleep health on cognitive function in HIV+ and HIVadults. J Int Neuropsychol Soc. 2018;24(10):1038-1046. doi:10. 1017/S1355617718000607

104. Gosselin N, De Beaumont L, Gagnon K, et al. BDNF Val66Met polymorphism interacts with sleep consolidation to predict ability to create new declarative memories. J Neurosci. 2016;36 (32):8390-8398. doi:10.1523/JNEUROSCI.4432-15.2016

105. Lee KA, Gay C, Portillo CJ, et al. Types of sleep problems in adults living with HIV/AIDS. J Clin Sleep Med. 2012;8(1):67-75. doi: $10.5664 /$ jcsm. 1666 
106. Stern Y. Cognitive reserve: implications for assessment and intervention. Folia Phoniatr Logop. 2013;65(2):49-54. doi:10.1159/000353443

107. Ward DD, Andel R, Saunders NL, et al. The BDNF Val66Met polymorphism moderates the effect of cognitive reserve on 36-month cognitive change in healthy older adults. Alzheimers Dement. 2017;3(3):323-331. doi:10.1016/j.trci.2017.04.006

108. Greenwood PM, Parasuraman R. Neuronal and cognitive plasticity: a neurocognitive framework for ameliorating cognitive aging. Front Aging Neurosci. 2010;2:150. doi:10.3389/ fnagi.2010.00150

109. Bachis A, Wenzel E, Boelk A, Becker J, Mocchetti I. The neurotrophin receptor p75 mediates gp120-induced loss of synaptic spines in aging mice. Neurobiol Aging. 2016;46:160-168. doi:10.1016/j.neurobiolaging.2016.07.001

110. Anand P, Springer SA, Copenhaver MM, Altice FL. Neurocognitive impairment and HIV risk factors: a reciprocal relationship. AIDS Behav. 2010;14(6):1213-1226. doi:10.1007/ s10461-010-9684-1

111. Collier AD, Haddow L, Brijkumar J, Moosa SM-Y, Benjamin L, Gupta KR. HIV cerebrospinal fluid escape and neurocognitive pathology in the era of combined antiretroviral therapy: what lies beneath the tip of the iceberg in Sub-Saharan Africa?. Brain Sci. 2018;8(10):190. doi:10.3390/brainsci8100190

112. Atluri VSR, Hidalgo M, Samikkannu T, Kurapati KRV, Nair M. Synaptic plasticity and neurological disorders in neurotropic viral infections. Neural Plast. 2015;2015:1-14. doi:10.1155/2015/ 138979

113. Olivier IS, Cacabelos R, Naidoo V. Risk factors and pathogenesis of HIV-associated neurocognitive disorder: the role of host genetics. Int $J$ Mol Sci. 2018;19(11):3594. doi:10.3390/ ijms 19113594

114. Tedaldi EM, Minniti NL, Fischer T. HIV-associated neurocognitive disorders: the relationship of HIV infection with physical and social comorbidities. Biomed Res Int. 2015;2015:1-13. doi:10.1155/2015/641913

115. Sun B, Abadjian L, Rempel H, Calosing C, Rothlind J, Pulliam L. Peripheral biomarkers do not correlate with cognitive impairment in highly active antiretroviral therapy-treated subjects with human immunodeficiency virus type 1 infection. J Neurovirol. 2010;16 (2):115-124. doi:10.3109/13550280903559789

116. Valcour V, Sithinamsuwan P, Letendre S, Ances B. Pathogenesis of HIV in the central nervous system. Curr HIV/AIDS Rep. 2011;8(1):54-61. doi:10.1007/s11904-010-0070-4

117. Saylor D, Dickens AM, Sacktor N, et al. HIV-associated neurocognitive disorder-pathogenesis and prospects for treatment. Nat Rev Neurol. 2016;12(4):234-248. doi:10.1038/nrneurol.2016.27

118. Ferrell D, Giunta B. The impact of HIV-1 on neurogenesis: implications for HAND. Cell Mol Life Sci. 2014;71 (22):4387-4392. doi:10.1007/s00018-014-1702-4
119. Shikuma CM, Nakamoto B, Shiramizu B, et al. Antiretroviral monocyte efficacy score linked to cognitive impairment in HIV. Antivir Ther. 2012;17(7):1233-1242. doi:10.3851/IMP2411

120. Mothobi NZ, Brew BJ. Neurocognitive dysfunction in the highly active antiretroviral therapy era. Curr Opin Infect Dis. 2012;25 (1):4-9. doi:10.1097/QCO.0b013e32834ef586

121. Míguez-Burbano MJ, Nair M, Lewis JE, Fishman J. The role of alcohol on platelets, thymus and cognitive performance among HIV-infected subjects: are they related? Platelets. 2009;20 (4):260-267. doi:10.1080/09537100902964759

122. Mogambery JC, Dawood H, Wilson D, Moodley A. HIVassociated neurocognitive disorder in a KwaZulu-Natal HIV clinic: a prospective study. South Afr J HIV Med. 2017;18 (1):1-5. doi:10.4102/sajhivmed.v18i1.732

123. Ene L. Human immunodeficiency virus in the brain-Culprit or facilitator? Infect Dis Res Treat. 2018;11:1178633717752687. doi:10.1177/1178633717752687

124. Kallianpur AR, Levine AJ. Host genetic factors predisposing to HIV-associated neurocognitive disorder. Curr HIV/AIDS Rep. 2014;11(3):336-352. doi:10.1007/s11904-014-0222-z

125. Nakku J, Kinyanda E, Hoskins S. Prevalence and factors associated with probable HIV dementia in an African population: a cross-sectional study of an HIV/AIDS clinic population. BMC Psychiatry. 2013;13(1):126. doi:10.1186/1471-244X-13-126

126. Valdez AN, Rubin LH, Neigh GN. Untangling the Gordian knot of HIV, stress, and cognitive impairment. Neurobiol Stress. 2016;4:44-54. doi:10.1016/j.ynstr.2016.02.005

127. Lam A, Mayo NE, Scott S, Brouillette M-J, Fellows LK. HIVrelated stigma affects cognition in older men living with HIV. JAIDS J Acquir Immune Defic Syndr. 2019;80(2):198-204. doi:10.1097/qai.0000000000001898

128. Namagga JK, Rukundo GZ, Voss JG. Prevalence and risk factors of HIV-associated neurocognitive disorders in rural Southwestern Uganda. J Assoc Nurses AIDS Care. 2019;30(5):531-538. doi:10.1097/JNC.0000000000000036

129. Paddick S-M, Flatt A, Eaton P, et al. 16 Prevalence and risk factors for HIV-associated neurocognitive impairment (hand) amongst adults aged 50 and over attending a HIV clinic in northern tanzania. J Neurol Neurosurg Psychiatry. 2017;88(8):A19LPA19. doi:10.1136/jnnp-2017-BNPA.40

130. Fazeli PL, Marquine MJ, Dufour C, et al. Physical activity is associated with better neurocognitive and everyday functioning among older adults with HIV disease. AIDS Behav. 2015;19 (8):1470-1477. doi:10.1007/s10461-015-1024-z

131. Debalkie Animut M, Sorrie MB, Birhanu YW, Teshale MY. High prevalence of neurocognitive disorders observed among adult people living with HIV/AIDS in Southern Ethiopia: a cross-sectional study. PLoS One. 2019;14(3):e0204636. doi:10.1371/journal.pone.0204636
Neuropsychiatric Disease and Treatment

\section{Publish your work in this journal}

Neuropsychiatric Disease and Treatment is an international, peerreviewed journal of clinical therapeutics and pharmacology focusing on concise rapid reporting of clinical or pre-clinical studies on a range of neuropsychiatric and neurological disorders. This journal is indexed on PubMed Central, the 'PsycINFO' database and CAS, an is the official journal of The International Neuropsychiatric Association (INA). The manuscript management system is completely online and includes a very quick and fair peer-review system, which is all easy to use. Visit http://www.dovepress.com/testimonials.php to read real quotes from published authors. 